\title{
Programação por Metas para Ajuste Não Linear
}

\author{
G. C. ALMEIDA ${ }^{1}$, L. W. MARCUCCI ${ }^{2}$, E. R. PINTO ${ }^{3}$, M. H. OLIVEIRA ${ }^{4 *}$, \\ H. O. FLORENTINO ${ }^{5}$, D. R. CANTANE ${ }^{6}$ e D. F. JONES ${ }^{7}$
}

Recebido em 12 de setembro de 2018 / Aceito em 11 de fevereiro de 2020

RESUMO. O objetivo deste trabalho foi comparar a eficácia dos modelos de Programação por Metas como ferramenta de regressão não linear, com os métodos de ajustes não lineares clássicos. Aplicou-se os modelos a dados experimentais de inativação de Salmonella spp. em carne moída suína. A investigação da eficiência dos métodos foi realizada pelo cálculo do erro absoluto.

Palavras-chave: regressão não linear, otimização por metas, Método de Mínimos Quadrados, LevenbergMarquardt.

\section{INTRODUÇÃ̃O}

A modelagem matemática e o ajuste de parâmetros podem ser utilizados nas ciências exatas com fins diversificados como, por exemplo, na engenharia de alimentos para estudar a ativação ou inativação de microrganismos nos alimentos, especialmente os de alta perecibilidade.

A microbiologia preditiva é uma área específica que usa modelos matemáticos para descrever a influência de fatores intrínsecos (atividade de água, $\mathrm{pH}$, etc) e extrínsecos (temperatura, umidade relativa da atmosfera, etc) no crescimento ou decrescimento de microrganismos nos alimentos.

\footnotetext{
*Autor correspondente: M. H. Oliveira - E-mail: marta.oliveira@unesp.br

${ }^{1}$ Pós-Graduação em Biometria, IB, UNESP/Botucatu - E-mail: gabriela.colovati@unesp.br https://orcid.org/00000002-1674-1554

${ }^{2}$ Pós-Graduação em Biometria, IB, UNESP/Botucatu - E-mail: leandro.marcucci@unesp.br https://orcid.org/00000002-6265-9439

3 Pós-Graduação em Biometria, IB, UNESP/Botucatu - E-mail: eduardo.pinto@unesp.br https://orcid.org/0000-00015057-6911

${ }^{4}$ Pós-Graduação em Biometria, IB, UNESP/Botucatu - E-mail: marta.oliveira@ unesp.br https://orcid.org/0000-00019047-7782

5 Departamento de Bioestatística, Biologia Vegetal, Parasitologia e Zoologia, IB, UNESP/Botucatu - E-mail: helenice.silva@unesp.br https://orcid.org/0000-0003-2740-8826

6 Departamento de Bioestatística, Biologia Vegetal, Parasitologia e Zoologia, IB, UNESP/Botucatu - E-mail: daniela.cantane@unesp.br https://orcid.org/0000-0001-5561-3929

${ }^{7}$ University of Portsmouth, Faculty of Technology, School of Mathematics \& Physics - E-mail: dylan.jones@ port.ac.uk https://orcid.org/0000-0002-9101-746X
} 
Essa metodologia permite fazer a descrição quantitativa da interferência, de cada fator observado, na vida de prateleira dos alimentos possibilitando previsões, redução de custos e melhoria na qualidade dos alimentos [2].

A referida metodologia pode ser uma importante ferramenta matemática em estudos para a descrição do crescimento de Salmonella spp., com relação ao tempo em produtos alimentícios. Neste trabaho enfatizou-se a aplicação destas técnicas ao estudo do crescimento de Salmonella spp. em carne moída suína, utilizada na fabricação de produtos cárneos embutidos. Os dados de logaritmo de unidade formadora de colônia (UFC) por grama em função do tempo (horas), à temperatura de $58^{\circ} \mathrm{C}$, foram obtidos no banco de dados Common Database for Predictive Microbiology (ComBase) [5,10]. Comparou-se os modelos de otimização Programação por Metas (PM) com os métodos clássicos: Método dos Mínimos Quadrados (MMQ) e de Levenberg-Marquardt (LM), já consolidados na literatura para o ajuste de funções não lineares que descrevem o decrescimento populacional de Salmonella spp. em carne moída suína. A verificação da eficiência dos métodos se deu pela análise do erro absoluto com as normas do máximo e da soma [3].

O termo Programação por Metas (do inglês Goal Programming) foi utilizado inicialmente por Charnes e Cooper, em 1961, tornando-se uma das principais ferramentas da otimização multiobjetivo [4]. Existe uma grande variedade de problemas de tomada de decisão que abrangem múltiplos objetivos, possibilitando a aplicação dos modelos PM em áreas de transporte, economia, engenharia e em muitos outros contextos [7].

A Programação por Metas procura a melhor solução de compromisso frente às metas estabelecidas no problema tratado. Para isto são incluídas no modelo matemático, que descreve o problema, restrições flexíveis associadas ao atendimento das metas estabelecidas. Este processo de modelagem faz uso de variáveis de desvios, as quais são relacionadas com as folgas entre os valores obtidos e o alvo a ser alcançado [9, 19].

Há diversas abordagens para PM, as quais podem ser encontradas em [15, 16, 17, 18]. Na Seção 2 deste trabalho serão apresentadas três destas abordagens: Programação por Metas Ponderada, Programação por Metas de Chebyshev e a Programação por Metas Estendida, visando a estimação de parâmetros em ajustes de funções não lineares.

\section{MÉTODOS}

A técnica de estimação de parâmetros para o ajuste de uma função $f$, sobre um conjunto de dados, é frequentemente apresentada na literatura como um problema de otimização na forma de mínimos quadrados. Em tais problemas, havendo um conjunto de dados disponíveis, escolhe-se um modelo matemático e busca-se minimizar a soma dos quadrados dos desvios, denominados resíduos, entre cada um dos pontos dados e a curva ajustada. O Método dos Mínimos Quadrados linear pode ser escrito na forma matricial dada por:

$$
A=\left(X^{t} X\right)^{-1}\left(X^{t} Y\right)
$$


em que $A$ é o vetor dos parâmetros ajustados, $X$ é a matriz dos termos independentes e $Y$ é o vetor dos termos dependentes. Essa expressão pode ser utilizada em ajustes não lineares desde que sejam feitas as linearizações necessárias, seja por meio de ferramentas analíticas ou numéricas. No entanto, essa é uma técnica limitada e pode ser melhorada pelo uso de ferramentas mais eficientes, como por exemplo, o método de regressão não linear de Gauss-Newton sintetizado na expressão:

$$
[\Delta A]=\left[J^{t} J\right]^{-1}\left[(-J)^{t} R\right],
$$

em que $\Delta A$ é o vetor coluna das diferenças dos parâmetros, $J$ é a matriz jacobiana da função $f$ a ser ajustada e $R$ é o vetor resíduo [3,13]. O método de Gauss-Newton tem forte convergência perto do mínimo, no entanto pode obter matrizes singulares, de forma a não convergir corretamente para o ponto esperado [1].

O método de Levenberg-Marquardt (LM) é outro método utilizado para resolver problemas de mínimos quadrados não lineares, foi publicado primeiramente em 1944 por Kenneth Levenberg [11] e aperfeiçoado por Donald Marquardt em 1963 [12]. Esse método concatena dois métodos clássicos de otimização: Gauss-Newton e Gradiente (máxima descida) [13]. O método de LM é iterativo, utiliza o método do Gradiente quando está distante do ponto esperado, e quando o valor obtido no ajuste está próximo do ponto ótimo é usado o método de Gauss-Newton [1].

No método LM é inserido um parâmetro $\mu$, positivo, na Equação (2.2). Este parâmetro ao ser multiplicado pela matriz identidade, acelera a convergência do método e/ou retira a singularidade da matriz no método de Gauss-Newton. Assim a Equação (2.2) pode ser reescrita na forma:

$$
[\Delta A]=\left[J^{t} J+\mu I\right]^{-1}\left[(-J)^{t} R\right] .
$$

A adição do fator $\mu I$ influencia na direção e no tamanho do passo, $\mu$ promove convergência quadrática do método de LM e deve ser atualizado a cada iteração [13]. O uso do método LM, em ajustes não lineares, deve ser feito após a linearização da função $f$, cujas informações são armazenadas na matriz jacobiana $J$.

Os métodos baseados na metodologia Programação por Metas possuem a vantagem de realizar o ajuste da função não linear na sua forma original (não requer a linearização) e de satisfazer um número predeterminado de metas e não apenas de uma simples função objetivo caracterizando um problema de otimização multiobjetivo.

\subsection{Programação por Metas Ponderada (PMP)}

A Programação por Metas é uma área da matemática que permite a modelagem de problemas de otimização nos quais $Q$ metas devem ser alcançadas. Para isto, define-se variáveis de desvios $\left(d_{i}^{+}\right.$ e $\left.d_{i}^{-}\right)$em torno dos alvos $y_{i}$ propostos $(i=1, \cdots, Q)$ de forma a estabelecer restrições flexíveis sobre as metas, permitindo a obtenção de valores acima ou abaixo dos valores alvos. O objetivo do modelo de otimização torna-se minimizar os desvios gerados em torno dos alvos. A forma 
estabelecida para minimização dos desvios determina a classificação da PM. A Programação por Metas Ponderada (PMP) estabelece pesos não-negativos $\left(u_{i}\right.$ e $\left.v_{i}\right)$ para cada um dos desvios $(i=1, \cdots, Q)$, e o objetivo torna-se a minimização da soma ponderada dos desvios [18].

De acordo com [9] a formulação algébrica da PMP, com $Q$ metas declaradas, é dada por:

$$
\begin{aligned}
& \text { Minimizar } \sum_{i=1}^{Q} \frac{u_{i} d_{i}^{-}}{\left|y_{i}\right|}+\frac{v_{i} d_{i}^{+}}{\left|y_{i}\right|} \\
& \text { Sujeito a } \quad f\left(x_{i}\right)+d_{i}^{-}-d_{i}^{+}=y_{i} \quad \text { para } \quad i=1, \cdots, Q \\
& x \in F \\
& d_{i}^{-}, d_{i}^{+} \geq 0, \quad \text { para } i=1, \cdots, Q
\end{aligned}
$$

no qual $F \subseteq \mathbb{R}^{Q}$ é a região viável composta de todos os pontos no espaço de decisão $x=$ $\left(x_{1}, \cdots, x_{Q}\right)$ em que $x_{i}, i=1, \cdots, Q$, são as váriáveis de decisão, $f\left(x_{i}\right)(i=1, \ldots, Q)$ são as funções, das variáveis de decisão associadas as metas $Q, y_{i}$ o alvo de cada meta $i, d_{i}^{-}$a variável de desvio negativo, $d_{i}^{+}$a variável de desvio positivo do alvo $i, u_{i}$ e $v_{i}$ os pesos preferenciais associados as variáveis de desvios da meta $i$, em que $i=1, \cdots, Q$. Os pesos são calibrados, pelo tomador de decisão (TD), mediante a análise da melhoria dos resultados obtidos com o auxílio de métodos de otimização.

Segundo Jones e Tamiz (2010), as divisões por $y_{i}$ na função objetivo são normalizações necessárias para redimensionalizar os respectivos desvios quando estes estiverem em diferentes unidades de medidas.

\subsection{Programação por Metas de Chebyshev (PMC)}

O modelo de Programação por Metas de Chebyshev (PMC), também conhecido como Programação por Metas Minmax (PM Minmax), é uma abordagem específica da Programação por Metas introduzida pela primeira vez em 1976 por Flavell [6]. O qual visa a minimização de um limitante superior $\lambda$ para os desvios.

Considerando uma relação contínua entre o desvio do alvo e seu peso associado, essa metodologia fornece uma solução equilibrada para o atendimento das diferentes metas. Assim, o propósito desse método é minimizar o pior desvio (o desvio máximo) dos alvos expressos [14]. 
A formulação algébrica da PMC com $Q$ metas definidas e $\lambda>0$ como sendo o limitante superior sobre os desvios é dada por:

$$
\begin{array}{lrl}
\text { Minimizar } & \lambda & \\
\text { Sujeito a } & f\left(x_{i}\right)+d_{i}^{-}-d_{i}^{+}=y_{i} \quad \text { para } \quad i=1, \cdots, Q \\
& d_{i}^{-} \leq \lambda & \text { para } \quad i=1, \cdots, Q \\
& d_{i}^{+} \leq \lambda & \text { para } \quad i=1, \cdots, Q \\
& & \\
& \lambda \in F & \\
& d_{i}^{-}, d_{i}^{+} \geq 0, &
\end{array}
$$

no qual as variáveis e parâmetros são definidos de forma similar aos apresentados no modelo PMP (2.4). Valores de $\lambda$ próximos a zero indicam que as metas definidas foram alcançadas de maneira balanceada [14].

\subsection{Programação por Metas Estendida (PME)}

O modelo de PME foi introduzido por Romero (2001) e nesta classificação de PM objetivase uma análise paramétrica entre eficiência e equilíbrio de alcance dos valores alvo das metas $[8,14,15]$.

Existem formas lexicográficas e não-lexicográficas do modelo, as quais usam a presença e ausência de ordenação lexicográfica de objetivos, respectivamente [8]. Neste artigo está sendo priorizado a aproximação por meio de uma curva aos dados reais fornecidos em vez de priorizar os objetivos, desta maneira a forma não lexicográfica é usada. A formulação algébrica da PME é dada por:

$$
\begin{aligned}
& \text { Minimizar } \quad \alpha \lambda+(1-\alpha) \sum_{i=1}^{Q} \frac{u_{i} d_{i}^{-}}{\left|y_{i}\right|}+\frac{v_{i} d_{i}^{+}}{\left|y_{i}\right|} \\
& \text { Sujeito a } \\
& f\left(x_{i}\right)+d_{i}^{-}-d_{i}^{+}=y_{i} \\
& \text { para } i=1, \cdots, Q \\
& d_{i}^{-} \leq \lambda \\
& \text { para } i=1, \cdots, Q \\
& d_{i}^{+} \leq \lambda \\
& \text { para } i=1, \cdots, Q \\
& x \in F \\
& \lambda \geq 0 \\
& d_{i}^{-}, d_{i}^{+} \geq 0 \text {, } \\
& \text { para } i=1, \cdots, Q
\end{aligned}
$$


no qual as variáveis e parâmetros são definidos de forma similar aos apresentados do modelo PMP (2.4) e $\alpha \in[0,1]$ : se $\alpha=0$ ou $\alpha=1$, temos a PMP ou PMC, respectivamente.

\subsection{Escolha dos pesos}

Jones e Tamiz (2010) descrevem um procedimento para escolha dos pesos envolvidos no processo de otimização para os métodos PMP, PMC e PME. De acordo com esses autores, todos os pesos devem ser não negativos e somente os pesos associados aos desvios não requeridos devem ser positivos. Os autores afirmam ainda que os pesos devem ser escolhidos pelo TD, mas quando este não estiver confiante quanto a esta escolha, pode-se utilizar alguma estratégia para auxílio nesta decisão, destacando-se que a interação desta estratégia com o TD é muito importante.

De acordo com [9], a citada interação pode ser realizada utilizando uma estratégia de mudanças no conjunto de pesos, ou parte deles, e reportar os resultados dos desvios das metas ao TD. Este processo necessita de um conjunto inicial de valores para os pesos, o qual pode ser obtido por valores estimados pelo TD, por valores iguais para todos os pesos ou por valores obtidos por um processo analítico de hierarquia, em que o TD estabelece uma ordem de importância para cada meta. A partir do conjunto inicial, é feita uma exploração no espaço dos pesos associados aos desvios não desejados. Para obter a mesma magnitude, em geral utiliza-se valores para os pesos cuja soma seja igual a 1. Jones e Tamiz (2010) propuseram uma heurística para guiar a exploração do espaço de pesos, objetivando analisar características que favoreçam a redução dos desvios e separaração de um conjunto contendo valores para os pesos que gerem diferentes soluções para o modelo de PM, isto permite que o TD possa escolher a que for mais conveniente ao seu interesse ou ao critério estabelecido por este.

Os problemas clássicos modelados utilizando PM, que em geral são problemas de otimização multiobjetivo, envolvem um número pequeno de metas (em geral até 5 metas). Este número reduzido de metas facilita a exploração do espaço de pesos e, portanto, viabiliza o uso da heurística proposta por Jones e Tamiz (2010). Mas a exploração do espaço de pesos, no presente problema, é dificultado devido a quantidade destes.

Desta forma, propõe-se um critério para escolha de pesos apropriados ao ajuste desejado. Neste critério, é dado um conjunto inicial de valores para os pesos e posteriormente resolve-se o modelo de PM desejado. O TD observa os desvios gerados e toma decisões no sentido de mudar o conjunto de valores dos pesos convenientes, de modo que sejam dados valores maiores aos pesos associados aos maiores desvios, os quais são os mais indesejáveis. Resolve-se o modelo de PM com este novo conjunto de pesos e repete-se este processo até obter valores de pesos aprovados pelo TD, baseado em alguma métrica para o erro.

\section{RESULTADOS COMPUTACIONAIS}

Os modelos apresentados foram aplicados a dados reais de decrescimento de Salmonella spp. em carne moída suína, obtendo, assim, dados do logaritmo de unidades formadoras de colônias 
(UFC) por grama no tempo (horas) sob uma temperatura de $58^{\circ} \mathrm{C}$, estes dados estão apresentados na Tabela 1.

Tabela 1: Dados do decrescimento de Salmonella spp. em carne moída.

\begin{tabular}{ccccccccc}
\hline$x_{i}=$ Tempo (h) & 0 & 0,08 & 0,25 & 0,33 & 0,42 & 0,50 & 0,67 & 0,83 \\
\hline$y_{i}=$ Log UFC/g & 7,64 & 6,99 & 5,61 & 4,82 & 3,89 & 3,00 & 2,06 & 1,48 \\
\hline
\end{tabular}

O ajuste da curva aos pontos da Tabela 1 foi realizado utilizando MMQ, LM e a abordagem de PM. Estes métodos foram implementados utilizando o software Octave e considerando a função não linear

$$
f(x)=a_{0} e^{a_{1} x}
$$

O propósito do ajuste é determinar valores para os parâmetros $a_{0}$ e $a_{1}$ tais que $f\left(x_{i}\right)=y_{i}$ para todo $i=1, \cdots, Q$, em que $x_{i} \in \mathbb{R}$ é o tempo (horas) e $y_{i}$ é o Log UFC/g conforme Tabela 1.

Para a implementação do MMQ, foi utilizada a Equação (2.1) considerando a linearização analítica da função $f$. Para o método de LM considerou-se a função lsqnonlin do software Octave com precisão máxima de $10^{-5}$, para norma do vetor solução e critério de parada de 7200 iterações. Os resultados destes ajustes estão mostrados na Tabela 3.

Para a aplicação dos modelos de PM considerou-se $Q=8$ metas a serem alcançadas, para os alvos $y_{i}$ a serem atingidos, ou seja, $f\left(x_{i}\right)=y_{i}$ para $i=1, \cdots, 8$. Para todos os métodos, exceto MMQ, foi utilizado o vetor nulo como estimativa inicial dos parâmetros de ajuste dos modelos $f\left(x_{i}\right), i=1, \cdots, 8$.

Para a escolha dos pesos apropriados ao ajuste da função (3.1), aos dados da Tabela 1, foi utilizado o critério proposto na subseção 2.4 , de forma que a soma destes seja igual a 1 , ou seja, $\sum_{i=1}^{8} u_{i}+v_{i}=1$. O TD, o grupo de autores, utilizou a métrica $\left|y_{i}-f\left(x_{i}\right)\right|$, com $i=1, \cdots, 8$, para estabelecer o conjunto de valores para os pesos utilizados nas PMP e PME. Os pesos, expostos na Tabela 2, foram definidos da seguinte maneira:

$$
\begin{aligned}
& \cdot f\left(x_{i}\right)-y_{i} \geq 0, \text { tem-se } u_{i}=10^{-4} \text { e } v_{i}=\frac{1}{Q}-10^{-4} \\
& \text { - } f\left(x_{i}\right)-y_{i}<0, \text { tem-se } u_{i}=\frac{1}{Q}-10^{-4} \text { e } v_{i}=10^{-4}
\end{aligned}
$$

A normalização nos modelos de PM foi desnecessária, pois os dados possuem a mesma unidade de medida, propiciando melhores ajustes. Utilizou-se $\lambda=0$ como condição inicial nos modelos PMC e PME e os resultados obtidos foram $\lambda=0,4191$ e $\lambda=0,4227$, respectivamente.

Na PME variou-se o valor de $\alpha$ no intervalo ]0,1[, com espaçamento $\Delta \alpha=10^{-2}$, observando que, a partir de $\alpha=0,05$ não ocorreram alterações significativas nos resultados alcançados. 
Tabela 2: Pesos dos modelos de PMP e PME.

\begin{tabular}{ccccccccc}
\hline$i$ & 1 & 2 & 3 & 4 & 5 & 6 & 7 & 8 \\
\hline$u_{i}$ & 0,0001 & 0,1249 & 0,1249 & 0,1249 & 0,1249 & 0,0001 & 0,0001 & 0,0001 \\
$v_{i}$ & 0,1249 & 0,0001 & 0,0001 & 0,0001 & 0,0001 & 0,1249 & 0,1249 & 0,1249 \\
\hline
\end{tabular}

Neste contexto, o TD escolheu como melhor valor $\alpha=0,05$ e os valores obtidos estão expostos na Tabela 3.

Na Tabela 3 estão apresentados os resultados para os parâmetros do ajuste $a_{0}$ e $a_{1}$ e para os erros na norma do máximo e na norma da soma (norma 1), encontrados nos modelos implementados. Nota-se que o pior ajuste foi obtido no MMQ e, baseado na norma 1, verifica-se que os modelos de PM apresentaram resultados melhores do que os métodos de ajuste de parâmetros clássicos.

Tabela 3: Parâmetros e erros da função de ajuste para todos os modelos.

\begin{tabular}{lccccc}
\hline & MMQ & LM & PMP & PMC & PME \\
\hline$a_{0}$ & 8,5473 & 8,0183 & 8,1273 & 8,0591 & 8,0433 \\
$a_{1}$ & $-2,0513$ & $-1,7770$ & $-1,8845$ & $-1,7596$ & $-1,7545$ \\
\hline Norma do Máximo & 0,9073 & 0,4678 & 0,5361 & 0,4191 & 0,4227 \\
Norma 1 & 2,6627 & 2,3583 & 2,3144 & 2,3542 & 2,3415 \\
\hline
\end{tabular}

Na Figura 1 estão ilustradas as curvas dos modelos descritos e, para melhor visualização, estas são apresentadas em tamanho ampliado na parte superior, pois não há diferença visual significativa dos ajustes encontrados. Para verificação da eficiência dos métodos é preciso fazer a análise dos erros encontrados.

\section{DISCUSSÃO DE RESULTADOS E CONCLUSÃO}

Para os dados experimentais utilizados no ajuste pode-se observar que os erros apresentados pelos modelos possuem mesma ordem de grandeza. No entanto, os erros obtidos pelos três modelos baseados na metodologia de Programação por Metas foram menores do que os erros dos métodos dos Mínimos Quadrados e de Levenberg-Marquardt, com excessão do PMP que encontrou um erro maior que o método de Levenberg-Marquardt para a norma do máximo. Os modelos de Programação por Metas não necessitam da linearização da função ajuste e encontraram resultados com precisões equivalentes ao método de LM.

Os resultados apontam que cada método de Programação por Metas atingiu seu objetivo. A PMP apresentou um erro menor que os demais modelos para a norma da soma, garantindo a minimização da soma ponderada dos desvios e a PMC obteve o menor erro máximo, o qual é limitante superior para o maior desvio dos alvos propostos, dentre todos os métodos implementados. A PME encontrou valores que mostram um equilíbrio entre os erros da norma 1 e da norma do máximo, dos métodos PMP e PMC, consolidando a concatenação dos métodos descrita na li- 


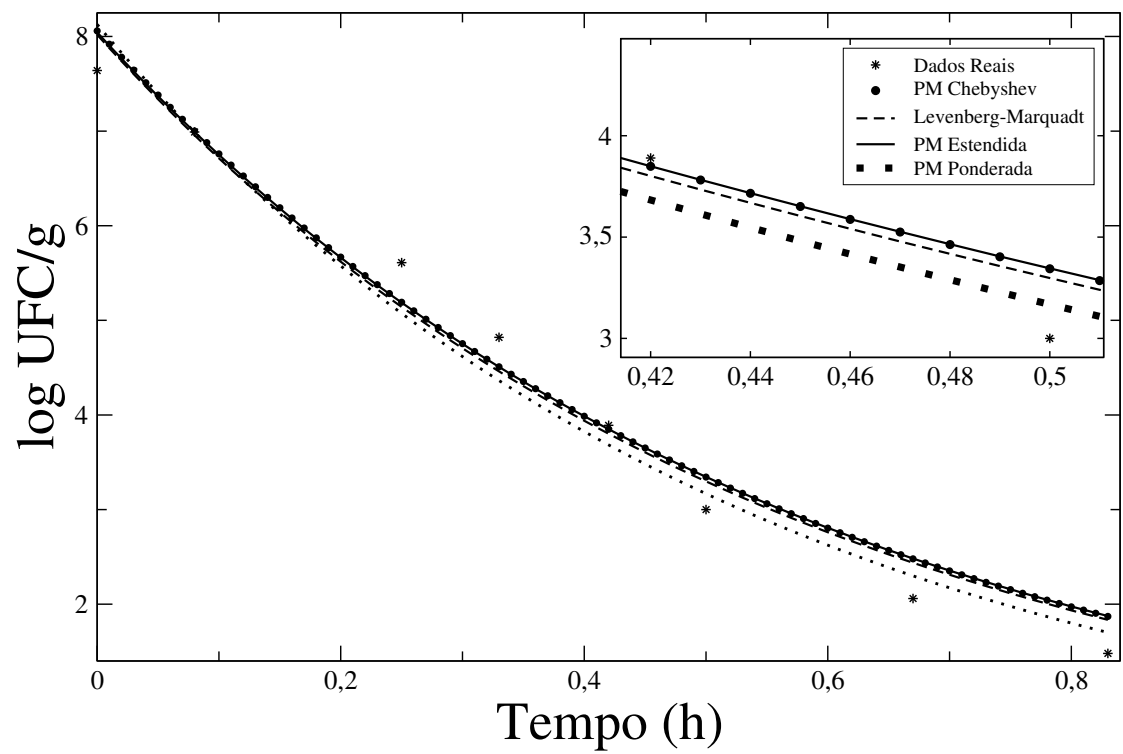

Figura 1: Comparação entre os dados reais e os modelos ajustados - Figura ampliada (parte superior).

teratura. A metodologia de Programação por Metas mostrou-se eficiente para as três abordagens utilizadas no trabalho.

Esta modelagem auxilia na resolução de problemas de otimização e pode fazer uso da programação existente em softwares gratuitos como, por exemplo, LibreOffice Calc e Octave.

\section{AGRADECIMENTOS}

O presente trabalho foi realizado com apoio da Coordenação de Aperfeiçoamento de Pessoal de Nível Superior - Brasil (CAPES) Número 88882.433353/2019-01 - Código de Financiamento 001; Conselho Nacional de Desenvolvimento Científico e Tecnológico (CNPq) Número 312551/2019-3; Fundação de Amparo à Pesquisa do Estado de São Paulo (FAPESP) Números 2013/07357-0, 2014/04353-8, 2015/07293-9 e 2019/05505-0; e Famat/UFU.

\footnotetext{
ABSTRACT. The aim of this work was to compare the effectiveness of Goal Programming models, as a nonlinear regression tool, with the classical nonlinear fitting methods. The models were applied to experimental data of inactivation Salmonella spp. in ground pork. The investigation of the methods' efficiency was made by calculation of the maximum and absolute errors.
}

Keywords: nonlinear regression, goal programming, Least Square Method, LevenbergMarquardt 


\section{REFERÊNCIAS}

[1] A.S. Benedito \& F.L.P. Santos. "A Novel Technique to Estimate Biological Parameters in an Epidemiology Problem". Springer, Cham (2017), pp. 112-122.

[2] L.S. Caixeta, M.F. Zotarelli \& M.H. Oliveira. Uso da microbiologia preditiva para a predição do crescimento microbiano em produtos cárneos embutidos. In "Anais do II Simpósio de Tecnologia e Ciência”. SimTeCi (2016), p. 43.

[3] S.C. Chapra \& R.P. Canale. "Numerical Methods for Engineers". McGraw-Hill Science/Engineering/Math (2009).

[4] A. Charnes \& W. Cooper. "Management Models and Industrial Applications of Linear Programming". John Wiley and Sons (1961).

[5] ComBase. SsJun_58_P11 (ID). Disponível em: https://www.combase.cc/index.php/en/. Acesso em: 03 julho 2018 (2018).

[6] R.B. Flavell. A new goal programming formulation. Omega, 4(6) (1976), 731-732.

[7] S. Gupta, A. Fügenschuh \& I. Ali. A Multi-Criteria Goal Programming Model to Analyze the Sustainable Goals of India. Sustainability, MDPI, Open Access Journal, 10(3) (2018), 1-19.

[8] D. Jones, H. Florentino, D. Cantane \& R. Oliveira. An extended goal programming methodology for analysis of a network encompassing multiple objectives and stakeholders. European Journal of Operational Research, 255(3) (2016), 845-855.

[9] D. Jones \& M. Tamiz. "Practical Goal Programming". Springer US (2010).

[10] V.K. Juneja, B.S. Eblen \& H.M. Marks. Thermal Inactivation of Salmonella Serotypes in Red Meat as Affected by Fat Content. Kluwer Academic Publishers, 2(3) (2000), 189-225.

[11] K. Levenberg. A method for the solution of certain non-linear problems in least squares. Quarterly of Applied Mathematics, 2(2) (1944), 164-168.

[12] D.W. Marquardt. An algorithm for least-squares estimation of nonlinear parameters. Journal of the society for Industrial and Applied Mathematics, 11(2) (1963), 431-441.

[13] J. Nocedal \& S.J. Wright. "Numerical Optimization". Springer (2006).

[14] C. Romero. Extended lexicographic goal programming: a unifying approach. Omega, 29(1) (2001), 63-71.

[15] C. Romero. A general structure of achievement function for a goal programming model. European Journal of Operational Research, 153(3) (2004), 675-686.

[16] A.F. Silva \& F.A.S. Marins. Revisão da literatura sobre modelos de Programação por Metas determinística e sob incerteza. Production, 25(1) (2015), 92-112.

[17] A.F. Silva, F.A.S. Marins, E.X. Dias \& R.C. Miranda. Fuzzy Goal Programming applied to the process of capital budget in an economic environment under uncertainty. Gest. Prod., São Carlos, 25(1) (2017), 148-159. 
[18] M. Tamiz \& D.F. Jones. A review of Goal Programming and its applications. Annals of Operations Research, 58 (1995), 39-53.

[19] N. Umarusman. Min-Max Goal Programming Approach For Solving Multi-Objective De Novo Programming Problems. International Journal of Operations Research, 10(2) (2013), 92-99. 